\title{
Managing clubfoot with Ponseti method: Our experience with children upto 3 years of age
}

\author{
Pawan Kumar Rawat ${ }^{1}$, Kunal Vij ${ }^{2, *}$ \\ Assistant Professor, Dept. of Orthopedics, Guru Ram Rai, Medical College Dehradun, Uttarakhand, India
}

*Corresponding Author: Kunal Vij

Email: drkunalvij@yahoo.in

\begin{abstract}
Introduction: Ponseti method has become the mainstay of congenital clubfoot treatment in children presenting at early age. The initial efficacy rate of Ponseti method reaches almost $100 \%$. The Ponseti method is also effective in older children with varied degree of success and preventing open joint surgery in almost every case. The Pirani method of evaluating the deformity and treatment outcome is easy and fairly reproducible and corresponds to the functional outcome of the treated feet.

Aim: The present study aims at elucidating the efficacy of Ponseti method of clubfoot correction in an inexperienced hand and evaluating the feasibility and worth of Pirani severity scoring system in deformity and treatment evaluation.

Materials and Methods: This is a prospective study carried out ii our institution Shri Guru Ram Rain Medical College Dehradun, India over a period of 2 1/2 years from June 2015 to December 2017 and includes 44 idiopathic Clubfeet corrective cast application at weekly interval as per Ponseti protocol and were assessed with Pirani scoring system. The cases were followed up regularly at least for 6 months.

Results: Following Ponseti protocol, we were able to achieve success rate of $95.45 \%$. We experienced only a few minor complications such as plaster sores and overcrowding of toes that too in initial phase of study.

Conclusion: Ponseti method is truly the gold standard of clubfoot management. Using Ponseti method, one can expect to achieve almost $100 \%$ success rate. It is also fairly effective in older children though with varied outcome or can at least prevent open joint surgery in them. The Pirani scoring system is clinical based, easy to apply and is in sync with Ponseti method.
\end{abstract}

Keywords: Clubfoot, Ponseti method, Pirani score.

\section{Introduction}

Clubfoot or congenital talipes equinovarus has plagued the human race since ages and has challenged the orthopaedic surgeon and the parents alike. Ponseti method of correction has revolutionized and has become the gold standard and mainstay in treatment of clubfoot over the past few decades. Dr Ignacio V. Ponseti described his path breaking technique of manual correction of clubfoot in year 1963 and further described in $1972^{1-3}$ with success rate as high as $98 \%$. His method gained popularity only in late nineties, when results of his subsequent long term studies reaffirmed his claim; and other orthopaedicians following his method, started reproducing these exciting results. ${ }^{3-6}$

Ponseti method is a sensible approach based on better understanding of the functional anatomy of the clubfoot and takes advantage of the logical response of young connective tissue and bone to manipulation and corrective casting. Ponseti was also sensible with his expectancy of results and aimed at achieving physiologically functional, supple, palntigrade, painfree and callus-free mobile foot rather than the cosmetically and radiologically appeasing one. Going by his nonsurgical method Ponseti et al, and subsequently other orthopaedic surgeons were able to avoid open joint surgery in as high as $97 \%$ of cases (Bor et $\left.\mathrm{al},{ }^{7}\right)$. The long term results of the treated feet were equally exciting and other centers were able to reproduce these. In 30 year follow-up study, cooper and Dietz found fairly comparable results in control and study groups. $78 \%$ of treated clubfoot had good or excellent results as compared to $85 \%$ in control group. ${ }^{8}$

Subsequently various methods of deformity evaluation and treatment monitoring were developed. Pirani scoring system is clinical based, easy to apply and is fairly reproducible with minor inter-observer variability.

The present study aims at elucidating the efficacy of Ponseti method of clubfoot correction in an inexperienced hand and evaluating the feasibility and worth of Pirani severity scoring system in deformity and treatment evaluation.

\section{Materials and Methods}

This is a prospective study conducted in our institution Shri Guru Ram Rai medical college Dehradun, India over a period of $21 / 2$ years from June 2015 to Dec 2017 we included 44 idiopathic clubfeet of 31 children of age group 2 weeks to 3 years.

\section{Inclusion Criteria}

1. All fresh cases less than 3 years of age with idiopathic clubfoot

2. Relapsed cases treated elsewhere earlier

3. Neglected cases that were treated elsewhere unsuccessfully.

\section{Exclusion Criteria}

1. Operated cases

2. Cases with other associated congenital and neurological malformations

3. Secondary or acquired clubfoot 
The deformity was assessed with Pirani severity scoring, podographic analysis, radiological analysis and serial photographs.

The cases were subjected to serial manipulation with corrective cast application at weekly interval as per Ponseti protocol.

Ponseti Method: The procedure described by Ponseti (1-2) can be divided into two phases-

1. Casting phase which consists of manipulation, casting and tenotomy.

2. Maintenance phase which is use of foot abduction brace to prevent relapse or recurrence.

In first few casts, cavus was corrected by supinating the forefoot by elevating the head of first metatarsal so as to align forefoot to the axis of hindfoot. In subsequent casts, forefoot adduction was corrected. Identifying the talar head, counter pressure is applied with thumb over its lateral aspect and forefoot is abducted and stretched to the limit of comfort of the child and held for at least 60 seconds. A good session of manipulation before casting is an essential and important step of deformity correction. A high groin cast is then applied maintaining the correction. Care is taken of proper molding and not to apply prolonged pressure over talar head to avoid plaster sore. The equinus was corrected last, only when anterior calcaneus along with midfoot and forefoot can be abducted underneath the talus and has been unlocked. This was often aided by percutaneous tenotomy of tendoachilles as an OPD procedure. The tibialis anterior tendon transfer to middle or lateral cuneiform can be conducted on relapsed cases with residual dynamic supination and adduction deformity. ${ }^{17-19}$
The corrective casts were applied weekly to the point where:

1. Full correction of deformities was achieved with $70-75^{\circ}$ of abduction and $15-20^{\circ}$ of dorsiflexion and child is able to actively dorsiflex the foot.

2. No further correction of deformities and improvement in Pirani score was achieved in 3 consecutive casts.

3. Number of casts exceeded 12 .

\section{Brace Application and follow-up}

The correction achieved is maintained in foot abduction brace which consists of well fitted high top shoes attached to a bar approximately equal to the shoulder width. The affected foot was held in $70^{\circ}$ and normal foot in $45^{\circ}$ of abduction. The child was made to apply the brace full time for initial 3 months than 14 hours daily (12 hours in night and 2 hours during day) for next 3-4 years. Strict compliance was ensured and parents were educated regarding its importance.

The child was followed up regularly fortnightly for initial 2 months and every month subsequently for a year.

\section{Pirani Scoring System}

Deformity assessment was done with Pirani scoring system. This system consists of 6 clinical scores having 3 grades (table: 1). It is simple, reproducible, acceptable universally, and has good inter-observer reliability. ${ }^{14-16}$ It assesses dynamic correction of the deformity and relies exclusively on clinical assessment and hence is in complete sync with Ponseti method of correction. Normal foot has score zero and severe deformity has score of 6 .

Table 1: Pirani scoring system

\begin{tabular}{|c|c|c|c|c|}
\hline \multicolumn{5}{|c|}{ Mid Foot Score } \\
\hline & & Normal & Moderate & Severe \\
\hline 1. & Curved lateral border & 0 & 0.5 & 1 \\
\hline 2. & Medial crease & 0 & 0.5 & 1 \\
\hline 3. & Talar head coverage & 0 & 0.5 & 1 \\
\hline \multicolumn{5}{|c|}{ Hind Foot Score } \\
\hline 4. & Posterior crease & 0 & 0.5 & 1 \\
\hline 5. & Rigid Equinus & 0 & 0.5 & 1 \\
\hline 6. & Empty heel & 0 & 0.5 & 1 \\
\hline \multicolumn{2}{|c|}{ Minimum score } & 0 & Maximum score & 6 \\
\hline
\end{tabular}

Periodic Podographs (foot impressions) were taken and food abduction deformity assessed with foot bimalleolar angle as by Jain et al. (Fig. 1). The normal foot bimalleolar angle is $82.5^{\circ}$.

The child's foot were radiographed at first visit and then at the end of the treatment. Various angles and relationships of various bones in relation to each other were made out and progress of treatment assessed by talo-calcaneal angle in antero-posterior and lateral views. (Fig. 2)

\section{Observations}

This is a prospective study conducted in our institution Shri Guru Ram Rai Medical College Dehradun, India over a period of $2 \frac{1}{2} 2$ years from June 2015 to Dec 2017, which includes 44 idiopathic clubfeet of 31 children of age group 2 weeks to 3 years. The cases were subjected to serial manipulation with corrective cast application at weekly interval as per Ponseti protocol. 
Maximum number of cases $(45.16 \%)$ presented before 6 months and about $70 \%$ cases presented before their first birthday. Only one case presented in the age group 30-36 months. The minimum age of presentation was 12 days and maximum age was 35 months. Male child were greater in number. 22 out of 31 were male and 9 female with sex ratio 2.44:1. Majority (58.06\%) were unilateral. Major bulk of patients $(67.74 \%)$ belonged to low socioeconomic group. Nearly all were fresh cases. Only one case was a relapse case, which had undergone complete treatment elsewhere. Familial preponderance was not found in any of the cases. Majority of the cases esp. those presenting late had tried some form of treatment mainly from unskilled local practitioner before seeking help. The mean number of casts applied was 6.45 . This number slightly improves to 5.82 if number of extra casts needed to treat relapsed cases is excluded. Mean number of casts needed in age group above $1 \frac{1}{1} 2$ year (mean 8.6 ) was slightly higher than the age group less than $1 \frac{1}{2} 2$ years (mean 6.18).

The mean initial Pirani score (PSi) was 3.86 which improved to final score (PSf) of 0.13 . The Pirani score after 3 months of follow-up (PS3m) was 0.31 because of relapse in 16 cases. With treatment, Pirani score after 6 months (PS6m) again improved to 0.27.

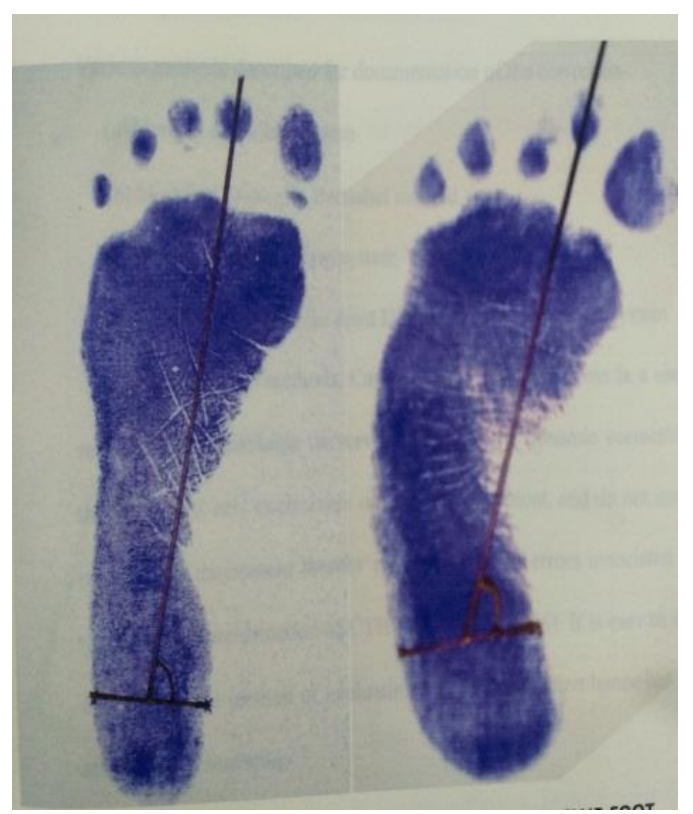

Fig. 1: Podogramic Evaluation: Foot Bimalleolar Angle (FBA) in normal and clubfoot
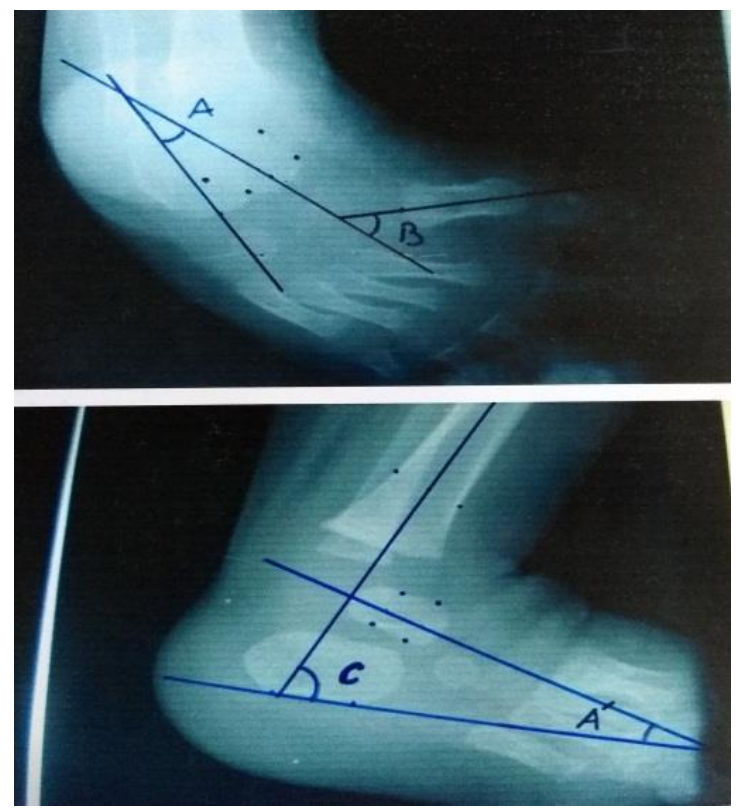

Fig. 2: Radiographic Evaluation; Angle A: Talocalcaneal angle in AP view; Angle B: Talus-1 ${ }^{\text {st }}$ Metatarsal angle; Angle C: Tibio-calcaneal angle; Angle A': Talocalcaneal angle in lateral view

Before treatment, majority (93.18\%) of cases had the Foot-bimalleolar angle (FBA) less than $70^{\circ}$ which got improved fairly to $>75^{\circ}$ in majority $(86.36 \%)$. The pre-treatment Talocalcaneal (TC) angle in AP view was between range $20-30^{\circ}$ in more than $84 \%$ of cases and post-treatment, majority got improved to $>30^{\circ}$ in almost $80 \%$ of cases. In lateral view, TC angle before treatment was in range of $10-20^{\circ}$ in majority $(72.72 \%)$; which after treatment improved to $>30^{\circ}$ in $81.18 \%$ of cases. The average pretreatment TC angle was $23.61^{\circ}$ in $\mathrm{AP}$ view and $15.78^{\circ}$ in lateral view which improved to $31.36^{\circ}$ and $32.08^{\circ}$ respectively. Talocalcaneal index increased from average $39.36^{\circ}$ to $63.44^{\circ}$.

Tendoachilles tenotomy was done in $31(70.45 \%)$ cases.

One case developed a minor complication of plaster sore over head of talus. Four $(9.09 \%)$ cases developed overcrowding of toes. Relapse rate after 3 months of follow-up was $36.36 \%$. Two feet could not be corrected even after 12 corrective casts and landed up in surgery, were considered as failures.

The overall success rate was $95.45 \%$. 


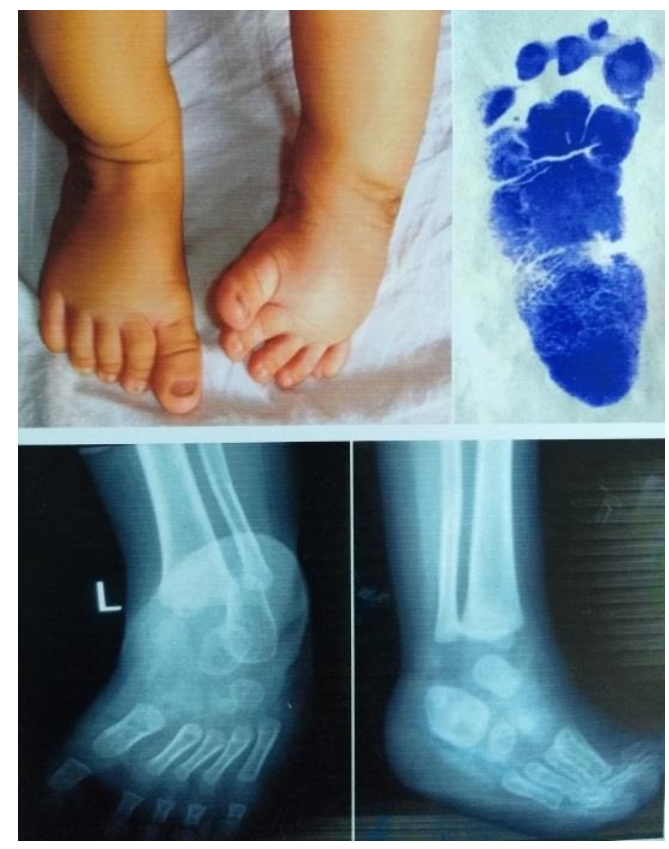

Fig. 3: Illustrated case pre-treatment

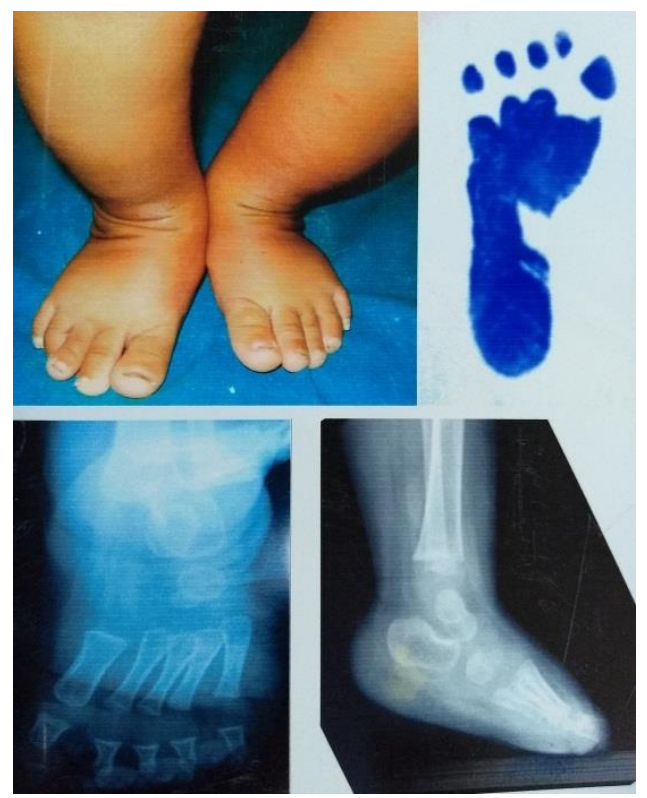

Fig. 4: Post-treatment

\section{Discussion}

More than $70 \%$ of the cases presented within first year of life among them nearly half of them presented in first 6 months. Many of them were taking treatment from some unskilled local practitioner. Though the cases presented quite late, but parents understood the gravity of the problem despite the fact that majority of cases belonged to low socio-economic status. There was a clear male predominance with the male to female ratio being 2.44:1 which was comparable to other studies such as Ponseti, 1963 (3:1), Cowell and Wein ${ }^{9}$ and Yamamoto. ${ }^{10}$ This increase in number of male child may be explained by hypothesis by Palmer ${ }^{11}$ that female child require greater number of predisposing factors to produce clubfoot deformity. Or it may be due to social stigma and bias towards girl child in our society and not getting treatment at all. Almost $2 / 3^{\text {rd }}$ of cases $(64.51 \%)$ were earlier treated somewhere else in one form or the other by all from a private practitioner to a quack. The main reason for leaving the treatment was dissatisfaction and cost of treatment.

The Pirani scoring system of deformity assessment and outcome documentation is easy and fairly correlates clinical appearance. It is simple, reproducible, acceptable universally, and has good inter-observer reliability. ${ }^{14-16}$ It assesses dynamic correction of the deformity and relies exclusively on clinical assessment and hence is in complete sync with Ponseti method of correction. More number of casts (average 8.6) were needed to fully correct the deformity in age group above 18 months than those who presented before 18 months (average 6.18) The number of casts needed to fully correct the deformity was 2-12 (average 6.45) which is slightly higher than the established studies such as 7.6 by Ponseti, ${ }^{1,2} 7$ by Laveg. ${ }^{3}$ The reasons could be larger age group, smallness of denominator. Here we included children up to 3 years of age. With increasing age, stubbornness of deformity to correction also increased. Excluding those 5 cases out of denominator the average number of casts needed dropped to 6.15. The average number of casts applied in those 5 cases who presented in later half of the study age group, was 8.6. This implies that Ponseti method is ideal for younger age group but has fairly good results in older age group also though this must be kept in mind that failure rate is appreciably higher. Another reason for higher number of casts could be inexperience as lesser number of casts was needed to achieve full correction in same age group in later half of the study. We encountered high rate of relapse $(36.36 \%)$. The main reason for relapse was noncompliance with bracing protocol. The parents were not putting the brace for adequate duration of time. Other causes included faulty splint application, broken splint and failure by the parents to be in regular follow-up or to change the brace with growth of child out of ignorance and poverty.

Two feet out of 44 were stubborn enough to not to get corrected fully even after 12 casts each with Pirani score 1.5 each at the end of the final cast. Barring these 2 cases all remained fully or almost fully corrected even after 6 months of follow-up. These cases eventually underwent open surgery. The overall success rate of $95.45 \%$ which is fairly encouraging enough and comparable to other studies- such as Ponseti, 2000 (98\%), Morcuende et al 2004 (98\%), Lehman et al 2003 (92\%), Tindall AJ et al (98\%), Kowalczyk B et al 2004 (88\%), Seger et al 2005 (94\%).

We encountered only a few minor complications in initial phase of the study. Only one patient developed plaster sore over the head of the talus. This was prevented subsequently by not applying prolonged 
pressure over the cast during molding and removing the dent over the talar head just in time while plaster is setting in. $9.09 \%$ of cases developed the over -crowding of the toes in initial phase of study, which was prevented later on by gently flattening of the cast antero-posteriorly so as to maintain transverse arch of foot. Soiling of plaster cast and consequent development of itching was prevented by encouraging the use of diaper. Parents were asked to remove the plaster cast at home itself by wetting it and bathing the child in the morning of the day of reapplication of cast to take care of the hygiene.

Tendoachilles tenotomy is an easy and good procedure to correct the remaining equinus in a single step. All these cases had intial Pirani score of 5 or more and can be used as a predictor of tenotomy. ${ }^{15}$ It is minimally invasive procedure and was performed on OPD basis without any complications on $70.45 \%$ of cases who still had Pirani score of 0 or 0.5 at midfoot and score of 1 at hindfoot. The remaining cases either did not need tenotomy or refused to go under the knife. Pirani et $\mathrm{al}^{13}$ carried out tenotomy in $90 \%$ of cases and Laveg et $\mathrm{al}^{3}$ performed in $78 \%$.

Radiography fairly correlates clinical appearance. The gradual increase in Talo-calcaneal angle in AP and lateral views, Tibio-calcaneal and Talus-first metatarsal angle fairly correlated with clinical appearance of the foot. However their utility can't be over-emphasized as our aim is a fully functional well aligned, cosmetically acceptable foot rather than anatomically perfectly aligned, scarred, painful foot as in surgery.

\section{Conclusion}

Ponseti method is truly the gold standard of clubfoot management. Using this method, one can expect to achieve almost $100 \%$ results even with relative inexperience such as us by making us move our thumb a few centimeters up and a few important minor modifications. Only minor hiccups such as noncompliance and consequent relapse with minor complications such as over-crowding of toes and plaster blister could not deter us keep using same treatment protocol. Though more successful in early age group, this method can be equally effective in older age group though with unpredictable outcome. Older and neglected cases with little hope can be subjected to this protocol and can get away with lesser invasive surgery without having to open the joint. Though Radiological assessment is helpful, but its utility can't be overemphasized as with Ponseti method, there is paradigm shift in in our understanding and assessment of deformity and our expectancy with treatment. Ponseti method is more focused on functional outcome rather than perfect anatomical alignment. Pirani scoring system of deformity evaluation and treatment documentation is easy and clinical based and is in complete sync with Ponseti method of clubfoot correction. The feasibility of podograhic analysis is questionable and has limited use. Educating the parents about deformity and winning their confidence is the key to ensure better compliance esp. with brace protocol. Educating the society, early detection and treatment of the deformity and ensuring regular follow up can all add to rectify the misery inflicted upon the innocents and having physiologically fully functional feet rather than leaving them scarred for life.

\section{Conflict of Interest: None.}

\section{References}

1. Ponseti IV, Smoley EN. Congenital club foot: the results of treatment. J Bone Joint Surg Am. 1963;45-A:261-344.

2. Ponseti IV, Campos J. Observations on pathogenesis and treatment of congenital club foot. Clin Orthop Relat Res. 1972;84:50-60. doi: 10.1097/00003086-19720500000011.

3. Laaveg SJ, Ponseti IV. Long-term results of treatment of congenital club foot. J Bone Joint Surg Am. 1980;62(1):23-31.

4. Cooper DM, Dietz FR. Treatment of idiopathic club foot: a thirty-year follow-up note. J Bone Joint Surg Am. 1995;77-A:1477-1489.

5. Morcuende JA, Abbasi D, Dolan LA, Ponseti IV. Results of an accelerated Ponseti protocol for club foot. J Pediatr Orthop. 2005;25:623-626. doi: 10.1097/01.bpo.0000162015.44865.5e.

6. Morcuende JA, Dolan LA, Dietz FR, Ponseti IV. Radical reduction in the rate of extensive corrective surgery for club foot using the Ponseti method. Pediatrics. 2004;113(2):376-380. doi: 10.1542/peds.113.2.376.

7. Bor N, Herzenberg JE, Frick SL. Ponseti management of club foot in older infants. Clin Orthop Relat Res. 2006;444:224-228. doi:

10.1097/01.blo.0000201147.12292.6b.

8. Cooper DM, Dietz FR. Treatment of idiopathic clubfoot: A thirty-year follow-up note. J Bone Joint Surg Am. 1995;77:1477-1489.

9. Cowell HR, Wein BK. Genetic aspect of clubfoot. J Bone Joint Surg Am. 1980;62(8):1381-1384.

10. Yamamoto H. A clinical, genetic and epidemiologic study of congenital clubfoot. Jinrui Idengaku Zasshi. 1979;24(1):37-44.

11. Palmer RM. Genetics of talipes equinus varus. $J$ Bone Joint Surg Am. 1964;46:542-556.

12. Scher DM, Feldman DS, Van Bosse HJ, Sala DA, Lehman WB. Predicting the need for tenotomy in the Ponseti method for correction of clubfeet. J Pediatr Orthop. 2004;24(4):349-352.

13. Pirani S, Zeznik L, Hodges D. Magnetic resonance imaging study of the congenital club foot treated with the Ponseti method. J Pediatr Orthop. 2001;21(6):719-726.

14. Flynn JM, Donohoe M, Mackenzie WG. An independent assessment of two club foot-classification systems. $J$ Pediatr Orthop. 1998;18(3):323-327.

15. Shaheen S, Jaiballa H, Pirani S. Interobserver reliability in Pirani club foot severity scoring between a paediatric orthopaedic surgeon and a physiotherapy assistant. $J$ Pediatr Orthop B. 2012;21(4):366-368. doi: 10.1097/BPB.0b013e3283514183.

16. Dyer PJ, Davis N. The role of the Pirani scoring system in the management of club foot by the Ponseti method. $J$ Bone Joint Surg Br. 2006;88(8):1082-1084. 
17. Bor N, Coplan JA, Herzenberg JE. Ponseti treatment for idiopathic club foot: minimum 5-year follow up. Clin Orthop Relat Res. 2009;467(5):1263-1270. doi: 10.1007/s11999-008-0683-8.

18. Eberhardt O, Peterlein CD, Fernandez FF, Wirth T. Midterm results of idiopathic club feet treated with the Ponseti method. Z Orthop Unfall. 2012;150(2):190-197. doi: 10.1055/s-0031-1298271.

19. 70. Church C, Coplan JA, Poljak D, Thabet AM,

Kowtharapu D, Lennon N, Marchesi S, Henley J, Starr R, Mason D, Belthur MV, Herzenberg JE, Miller F. A

comprehensive outcome comparison of surgical and Ponseti club foot treatments with reference to pediatric norms. J Child Orthop. 2012;6(1):51-59. doi: 10.1007/s11832-012-0387-1.

How to cite this article: Rawat PK, Vij K. Managing clubfoot with Ponseti method: Our experience with children upto 3 years of age. Indian J Orthop Surg. 2018;4(3):290-295. 